\title{
Optical impedance matching with scanning near-field optical microscopy (SNOM)
}

\author{
A Gademann, ${ }^{1}$ I V Shvets, ${ }^{1}$ and C Durkan ${ }^{2}$ \\ ${ }^{1}$ SFI Laboratory, Physics Department, Trinity College, Dublin 2, Ireland \\ ${ }^{2}$ University of Cambridge, Department of Engineering, \\ Trumpington Street, Cambridge CB2 1PZ, UK
}

(Dated: January 22, 2003)

We present an experimental study of the coupling of light from a waveguide into a submicron size optical transmission line. We demonstrate that the coupling efficiency depends upon the polarization direction in the waveguide. On the condition when a quasi TEM mode is launched in the transmission line we observe a substantial enhancement of the coupling efficiency. We demonstrate that the coupling efficiency also depends on the mismatch of the impedances of waveguide and transmission line, that is in turn determined by their respective geometries. We have made a prediction of the condition of the maximum coupling efficiency through the approach of impedance matching. We believe these results can be of importance in the development of new concepts of probes for scanning near-field optical microscopy. 


\section{INTRODUCTION}

A clear distinction is made between transmission lines and waveguides in microwave theory [1]. Transmission lines consist of two or more conductors separated by a dielectric. They support TEM or quasi-TEM modes, without any cut-off frequency.

Waveguides, on the other hand, are generally represented by a metal tube of circular, rectangular or other cross-section filled with a dielectric. They cannot support a true TEM mode, in which both field vectors $\mathrm{E}$ and $\mathrm{H}$ are perpendicular to the propagation direction. Instead they support $T E_{m n}$ or $T M_{m n}$ modes with only electric or magnetic field vector perpendicular to the waveguide axis respectively. Each mode of a waveguide is characterized by its cut-off frequency, i.e. the frequency below which the mode cannot propagate without very high attenuation. The dominant mode, i.e. the mode with the lowest cut-off frequency, is usually employed to transmit electromagnetic waves in a waveguide. In the case of a circular waveguide, the most relevant to the experiment discussed below, the cut-off frequency of the dominant $T E_{11}$ mode is

$$
f_{c}=\frac{1.841 \cdot c}{2 \cdot \pi \cdot r_{0}}
$$

where $r_{0}$ is the radius of the waveguide. This formula is valid for a waveguide with perfectly conducting walls. Here we assumed that dielectric and magnetic constants $\epsilon$ and $\mu$ are both equal to 1 . If the walls are made out of a real metal with nonzero resistance, the cut-off frequency is less well-defined [2]

The coupling efficiency of electromagnetic waves from waveguides to transmission lines depends upon the polarization direction in the waveguide with respect to the transmis-

sion line axis [3], and on the characteristic impedances of both structures. The concept of 
impedance matching is commonly used in the radio- and microwave frequency range. In the optical frequency range impedance matching is much more difficult to demonstrate due to the submicron size and the difficulties with fabricating of the optical structures required for such experiments. The notion of optical impedance is rarely used.

The prime purpose of the present study was to demonstrate experimentally that these fundamental principles hold for the electromagnetic waves in the optical regime. We performed the experiment using Scanning Near-Field Optical Microscopy (SNOM) [4], a technique which allows resolution well below the diffraction limit. The key element of the technique is a probe, usually consisting of a tapered optical fiber coated with metal so to form an aperture of the size 30 to $150 \mathrm{~nm}$ at the end. The tapered end of the probe coated with metal works as an overdamped waveguide since the wavelength is greater than $\lambda_{c}$. In this paper we report polarization dependent coupling of light from such a waveguide into a coplanar transmission line with a subwavlength separation between the conductors.

The advantage of using SNOM for such an experiment is that on can easily draw parallels between SNOM probes and microwave waveguides. Besides, one could bring the SNOM probe to a desired location on a sample containing transmission lines of known impedance and thus a number of experiments on impedance matching could be performed using the same probe. It is well appreciated that more commonplace acceptance of SNOM as a measurement technique depends on development of new probes capable of better resolution and more straightforward image interpretation. The work on new SNOM probes is paid much attention by the near-field optics community $[5,6]$.

Therefore the second purpose of the present study was to establish what implications impedance matching concepts may have for development of novel SNOM probes. 


\section{EXPERIMENT}

The instrument used for the experiment is a reflection-mode SNOM. The collection of the light is performed by an elliptical mirror [7-9]. The probe/sample interface is located at the first focal point, while the light is collected by a photo multiplier tube located at the second focal point. The optical layout can be seen in figure 1.

The probes are produced by tube etching proposed by Stockle et al [10]. They are coated to have $\sim 40 \mathrm{~nm}$ thick aluminum film at the tip apex forming an aperture at the very end of the tip. The laser used is a $635 \mathrm{~nm}$ diode laser. The light coming from the tip can be polarized by using a quarter waveplate and a half waveplate. The polarization is measured by placing an analyzer in between the probe and the PMT and it could be chosen in the range of 1:1 to 1:20 with alignment along any desired direction.

Two types of samples were produced by e-beam lithography. They consisted of gold lines, $20 \mathrm{~nm}$ thick and $1000 \mathrm{~nm}$ wide, formed on a silicon substrate. The distance between the lines is $250 \mathrm{~nm}$ for sample 1 and varies regularly between $200 \mathrm{~nm}$ to $1000 \mathrm{~nm}$ for sample 2 . The area between the lines is the bare silicon substrate.

Due to the high confinement of light in the aperture region of the probe, a distance feedback mechanism is required to maintain the sample within the near-field of the aperture. The technique used here was shear-force detection $[11,12]$, which allows us to simultaneously (1) image the sample topography and (2) obtain SNOM images. The probe could be kept at a constant height of 5 to $30 \mathrm{~nm}$ above the sample, with the accuracy of $\pm 2 \mathrm{~nm}$ using the shear-force feedback system described in [13]. 


\section{RESULTS}

Figure 2 shows two optical SNOM images of a $4.7 \mu m \times 4.7 \mu m$ area of sample 1 . Figure $2 \mathrm{a}$ is recorded with light polarized parallel to the Au-lines (TM polarisation) and figure $2 \mathrm{~b}$ with light polarized perpendicular to the lines (TE polarization). Analyzer was not used in these experiments. Particles of dust identifiable on both images indicated that they were taken at the same area of the sample. Figure 2 a shows the line as bright and the gap as dark. This was expected due to the higher reflectivity of Au compared to Si and also due to the elevation of the Au-lines above the Si substrate. The Au-film thickness results in increased shadowing of reflected light in between the lines. Figure $2 \mathrm{~b}$ in contrast shows the lines as dark and the gap as bright. Comparing the actual measured intensities in the middle of the Au-lines shows that a small change of less than $10 \%$ can be detected between the two directions of polarization. Whereas if the values measured in the gaps are taken, a change of polarization results in an intensity change of up to $80 \%$. This can be seen in the profiles (figure 3) taken at the representative cross-section AA' of figure 2.

Figure 4 shows topography and the optical SNOM images of a $9.3 \mu \mathrm{m} \times 9.3 \mu \mathrm{m}$ area of the second sample. Figure $4 \mathrm{a}$ is the shear-force image of the sample and $4 \mathrm{~b}$ and $4 \mathrm{c}$ are the optical images with the main axis of polarization parallel and perpendicular to the lines respectively. The same dust particles identifiable on all three images indicate that the scans are all taken at the same area. The tip used for this experiment had an approximate aperture of $150 \mathrm{~nm}$, as confirmed by Scanning Electron Microscope (SEM) for probes produced in the same way. Analyzer was not used in this experiment. Optical results presented in figures 2 and 4 with the same direction of polarization compare well. Once the polarization direction is set perpendicular to the lines (TE polarization) an increase in signal intensity in the gaps 
between the gold lines is observed compared to the images measured with parallel polarized light (TM polarization). Figure 5 shows this in the representative cross-section. However, there is an additional feature in the result shown in figure 4. As the gap is getting wider from $200 \mathrm{~nm}$ to $400 \mathrm{~nm}$, the intensity of the optical signal measured at the gap intensity increases and then with further increase in the gap width, it decreases provided the polarisation is TE.

Figure 6 shows topography and optical SNOM images of a $9.3 \mu \mathrm{m} \times 9.3 \mu \mathrm{m}$ area of the second sample. Figure 6 a shows the shear-force image of the sample and $6 \mathrm{~b}$ shows the optical image taken with polarization perpendicular to the gold lines (TE). The same dust particles identifiable on both images indicate that the scans are taken at the same area, they can also be identified in the images of figure 4 showing the relation between the images. The tip used for this scans had an aperture larger than the one used for the previous scans. It was in the range of $180-200 \mathrm{~nm}$. Comparing figures $4 \mathrm{c}$ and $6 \mathrm{~b}$ shows the same general characteristics of a higher intensity in the gaps and a change of intensity depending on gap width provided that TE polarization was used. In figure $6 \mathrm{~b}$ though the maximum intensity is observed when the gap width is $600 \mathrm{~nm}$ compared to $400 \mathrm{~nm}$ in figure $4 \mathrm{c}$.

\section{DISCUSSION}

These results can be explained noting that each pair of $\mathrm{Au}$ lines form a coplanar transmission line. It can be modeled as coplanar strip transmission line (figure 8a) or a coplanar waveguide (figure 8b). The analytical expression for the impedance $Z_{0}[14]$ for the coplanar stripline is 


$$
Z_{0}=\frac{120 \cdot \pi}{\sqrt{\epsilon_{e}}} \frac{K(k)}{K\left(k^{\prime}\right)}
$$

Impedance of the coplanar waveguide is

$$
Z_{0}=\frac{30 \cdot \pi}{\sqrt{\epsilon_{e}}} \frac{K\left(k^{\prime}\right)}{K(k)}
$$

Here $\epsilon_{e}$ is the effective dielectric constant, that is dependent on the dielectric constant of the substrate and the dimensions of the structures. $K(k) / K\left(k^{\prime}\right)$ is only dependent on the dimensions of metal structures.

Using these expressions for the dimensions of sample 1 predicts an impedance of $106 \Omega$ or $64 \Omega$ using formulas (2) and (3) respectively. Table I shows the prediction for sample 2 taking formulas (2) and (3). Due to the nature of the sample used, consisting of an array of parallel lines, none of the two models describes the impedance value accurately. This can be seen in figure 8. While coplanar stripline consists of two parallel lines without any other conductor in the vicinity, coplanar waveguide consists of one line with infinitely large planes left and right of it. Therefore the real impedance value should be in the range between the values calculated using formulas (2) and (3).

When the electric field from the tip is perpendicular to the line (TE polarization), a quasi TEM mode can be excited in the transmission line. This is the dominant mode of this structure and does not have the limitation of a cut-off frequency. It results in an enhanced coupling of energy from a probe into the line structure. When the electric field from the probe is parallel to the lines (TM polarization) no quasi TEM mode can be excited. In the first case light gets through subwavelength size aperture of the SNOM probe more efficiently and in the second, its greater fraction is reflected back into the probe.

A mode similar to $T E_{11}$ is expected to be excited at the output of the probe. An analytical 
expression for the impedance [1] is

$$
Z_{g}=\eta \frac{\lambda}{\lambda_{g}}
$$

with $\eta=\sqrt{\frac{\mu}{\epsilon}}$ being the intrinsic impedance and $\lambda_{g}$ the guide wavelength. Using formula 4 predicts an impedance value of $83 \Omega$ for a tip with an aperture of $150 \mathrm{~nm}$. Larger aperture sizes result in larger impedance values.

Figure 4c shows a maximum intensity at the 400nm gap size for TE polarization. Taking this result and comparing it to table I shows that for a gap with $400 \mathrm{~nm}$ width the impedance values are $120 \Omega$ or $73 \Omega$ depending on the model used. This suggests that the impedance value of the sample is closer to the one of the coplanar waveguide model which is intuitively clear.

Figure 6 shows the maximum intensity at the 600nm gap size. Table I suggests an impedance value of $133 \Omega$ or $82 \Omega$. As impedance is greater at $600 \mathrm{~nm}$ separation than at $400 \mathrm{~nm}$, one can expect that the impedance of the SNOM probe in figures 6, 7 is greater than in figures 4,5 . This is consistent with our indication that the probe in figures 6,7 had a greater aperture. Using the values of table one we can estimate a $25 \%$ larger aperture.

\section{CONCLUSIONS}

We have presented an experimental study of light coupling from a circular waveguide into a submicron size optical transmission line. We demonstrated that the coupling efficiency depends on the direction of polarization and on the condition when a quasi TEM mode is launched in the transmission line we observe a substantial enhancement of the coupling efficiency. We have made a prediction for the condition of the maximum coupling efficiency 
through the approach of impedance matching. By using a sample with various impedance values imaged with probes of different apertures we demonstrated that these predictions hold well at least semi-quantitatively.

We believe these results can be of importance in development of new concepts for high resolution optical imaging by means of SNOM. Samples with variable separation between the lines could be used as test samples to identify the size of the aperture at the tip. It also shows that results depend on tip (polarization, extinction ratio and aperture size), type of sample (transmission line structures) and the interaction between both. Possibly this could lead to a probe that is not designed as a waveguide but rather as a submicron transmission line, which could provide better extinction ratios.

\section{Acknowledgements}

We would like to acknowledge the funding by the EU under the TMR-project NanoSNOM (ERBFMRXCT98-0242), Magnetude Project (G5RD-CT-1999-00005) and by Science Foundation Ireland. 


\section{References}

[1] Sander K F 1987 Microwave Components and Systems (Addison-Wesley Publishing Inc.)

[2] Novotny L and Hafner C Phys. Rev. E 50 4094-4106 (1994)

[3] Chang K 1989 Handbook of Microwave and Optical Components vol 1 (J Wiley \& Sons)

[4] Pohl D W, Denk W and Lanz M Appl. Phys. Lett. 44 651-653 (1984)

[5] Naber A, Molenda D, Fischer U C, Maas H J, Hoppener C, Lu N and Fuchs H Phys. Rev. Lett. 89 art. nr. 210801 (2002)

[6] Bouhelier A, Toquant J, Tamaru H, Guntherodt H J, Pohl D W and Schider G Appl. Phys. Lett. 79 683-685 (2001)

[7] Durkan C and Shvets I V J. Appl. Phys. 83 1171-1176 (1998)

[8] Durkan C and Shvets I V Ultramicroscopy 61 227-231 (1995)

[9] Durkan C and Shvets I V J. Appl. Phys. 83 1837-1843 (1998)

[10] Stockle R, Fokas C, Deckert V, Zenobi R, Sick B, Hecht B and Wild U P Appl. Phys. Lett. 75 160-162 (1999)

[11] Betzig E, Finn P L and Weiner J S Appl. Phys. Lett. 60 2484-2486 (1992)

[12] Toledo-Crow R, Yang P C, Chen Y and Vaez-Iravani M Appl. Phys. Lett. 60 2957-2959 (1992)

[13] Kantor R, Lesnak M, Berdunov N and Shvets I V Appl. Surf. Sci. 145 510-513 (1999)

[14] Chang K 1989 Handbook of Microwave and Optical Components vol 1, chapter 1.3 (J Wiley \& Sons) 
FIG. 1: Schematic of the optical layout of the reflection mode SNOM.

FIG. 2: Scans of the same area of the sample taken with polarization along the transmission line structures (TM polarization) (a) and perpendicular to them (TE polarization) (b). The polarization directions used for the respective image are indicated by arrows.

FIG. 3: Representative cross-section taken at the lines AA' marked in figures 2a and 2b. 
TABLE I: Impedances for sample 2.

\begin{tabular}{c|c|c} 
Gap width, & Coplanar Stripline & Coplanar Waveguide \\
nm & Impedance, $\Omega$ & Impedance, $\Omega$ \\
\hline 200 & 100 & 60 \\
300 & 111 & 67 \\
400 & 120 & 73 \\
500 & 127 & 77 \\
600 & 133 & 82
\end{tabular}


FIG. 4: Scans of the same area of sample 2. (a) shear force topography image. (b) and (c) optical images taken with polarized light along the lines and perpendicular to the lines respectively. The polarization direction used for the respective image is indicated in the two optical images by arrows.

FIG. 5: Representative cross-sections taken at the lines BB' marked in figure 4 for TM polarization (5a) and TE polarization (5b).

FIG. 6: Scans of the same area of sample 2. (a) shear force topography image and (b) an optical images taken with light polarized perpendicular to the lines. 
FIG. 7: Representative cross-sections taken at the lines CC' marked in figure 6

FIG. 8: Sketch of cross-sections of a single coplanar stripline (a) and a single coplanar waveguide (b). Coplanar stripline consists of two parallel individual lines. Coplanar waveguide consists of a single line positioned in between two infinitely large metal areas left and right of it. The two areas have their edges parallel to the line. 


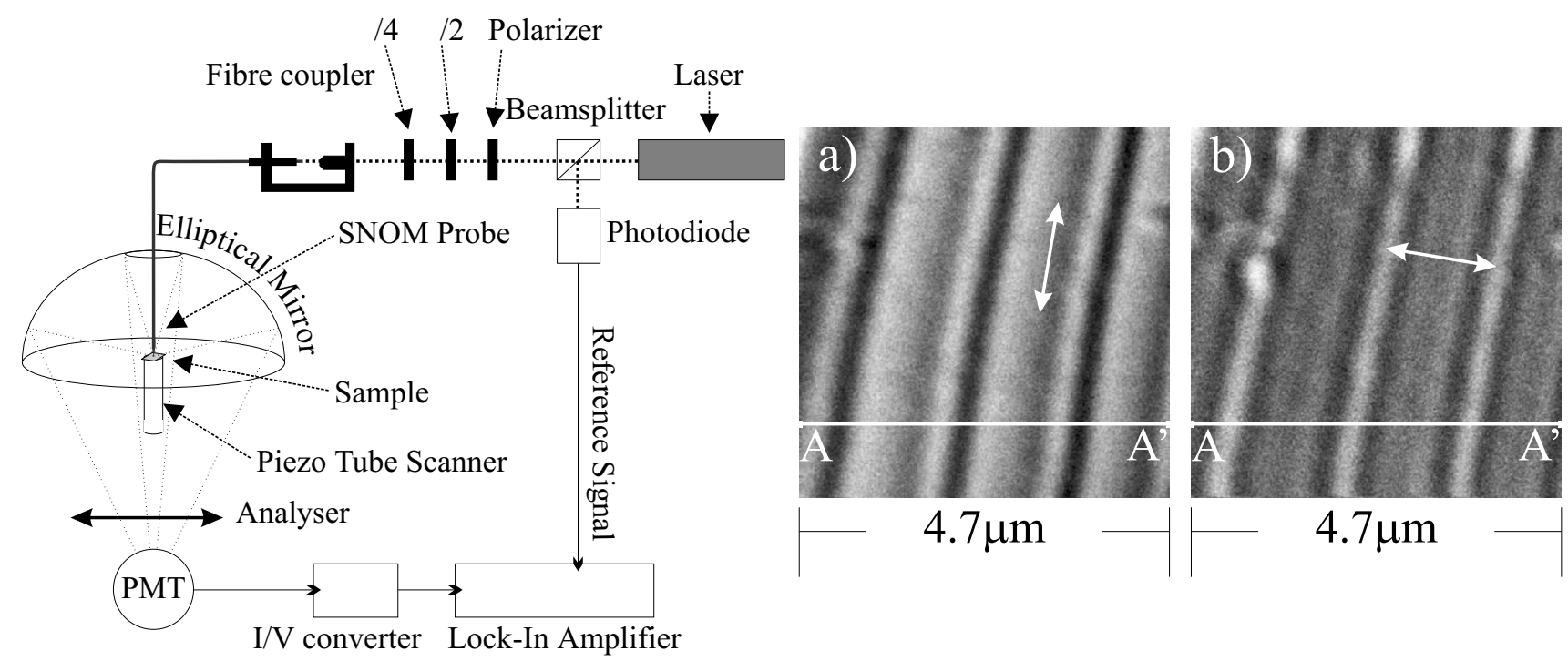

Figure 1

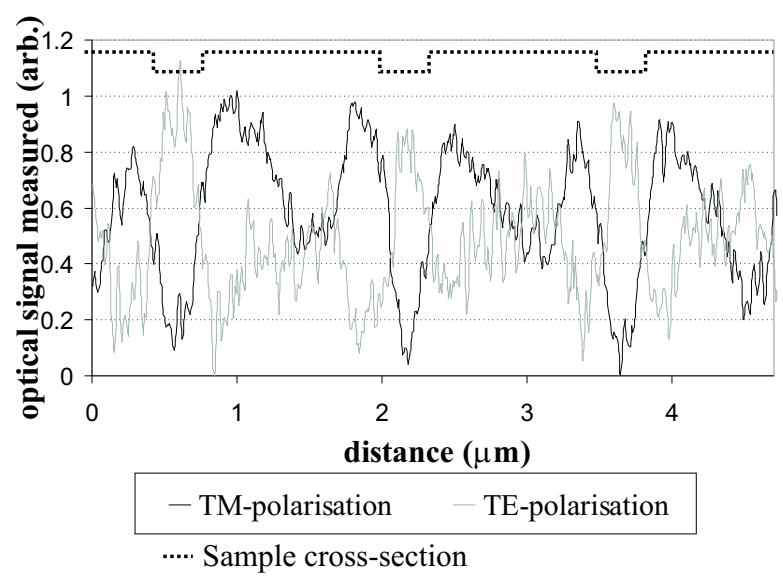

Figure 2
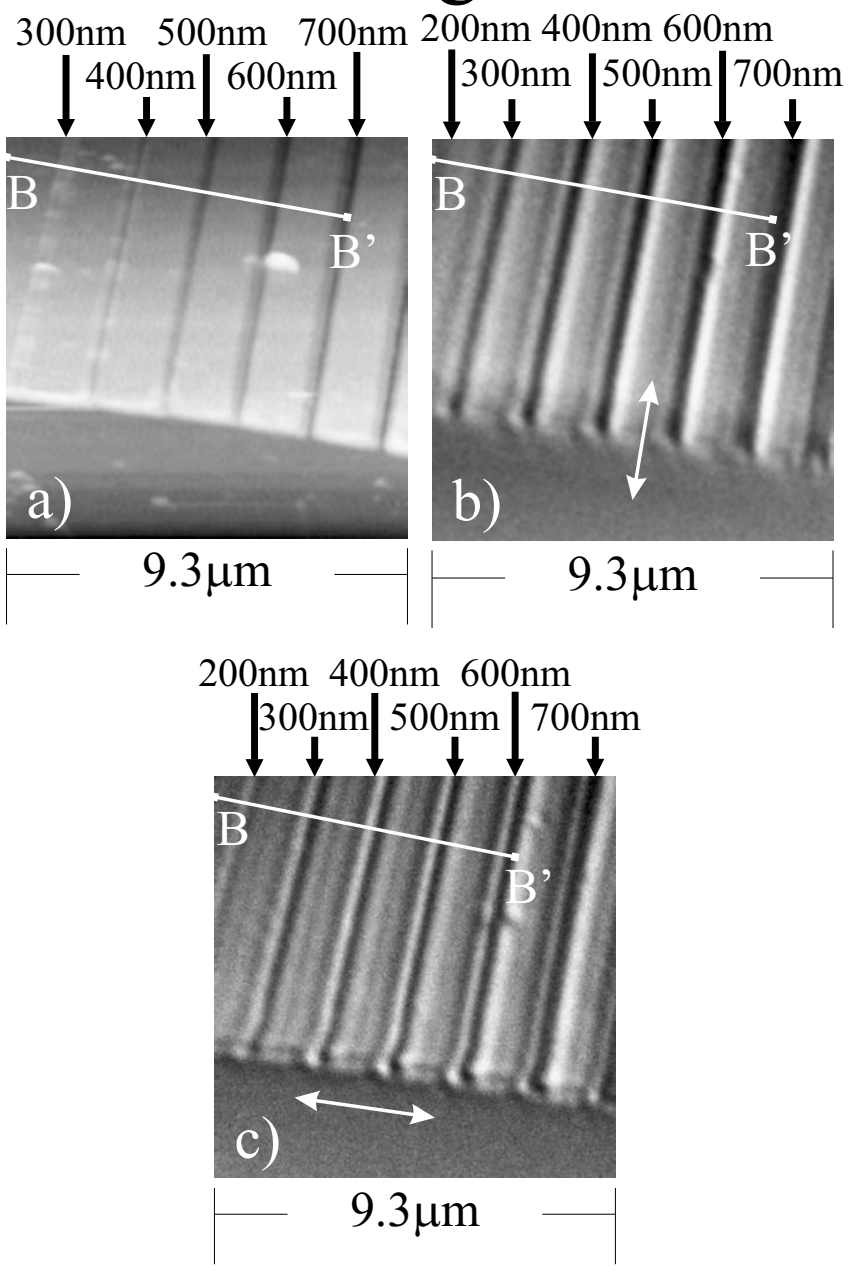

Figure 3

Figure 4 
a)

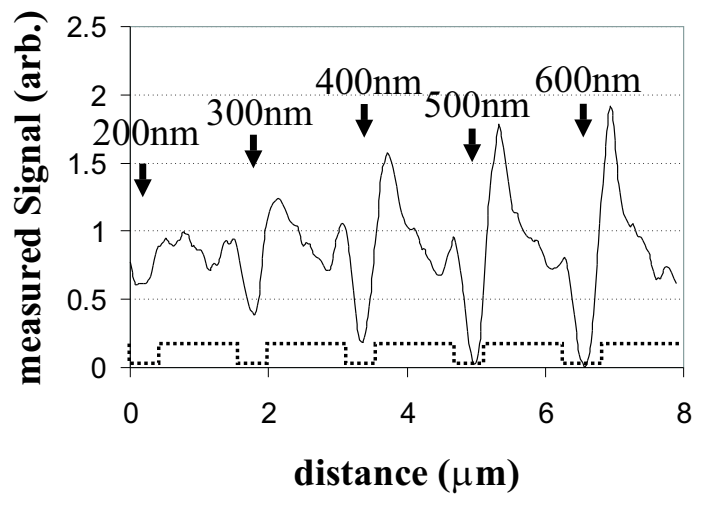

- TM-polarisation

.....Sample Cross-section

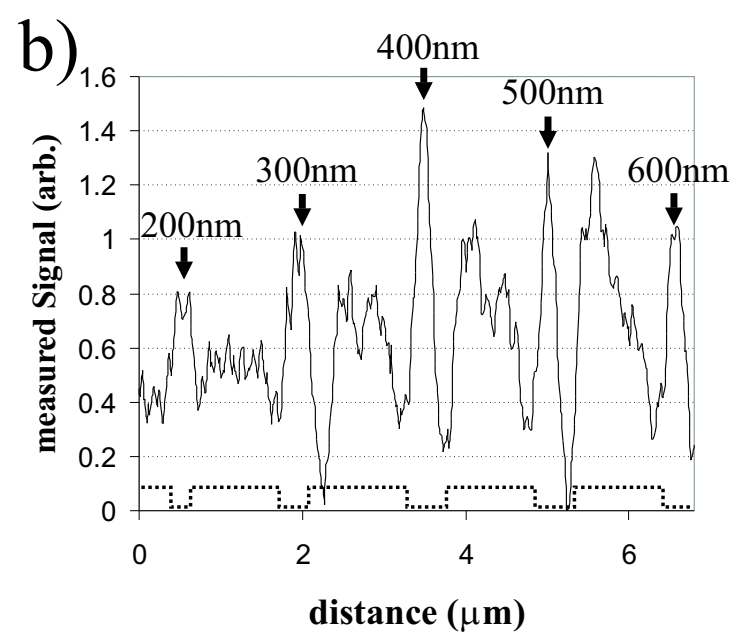

- TE-polarisation

....'Sample Cross-section

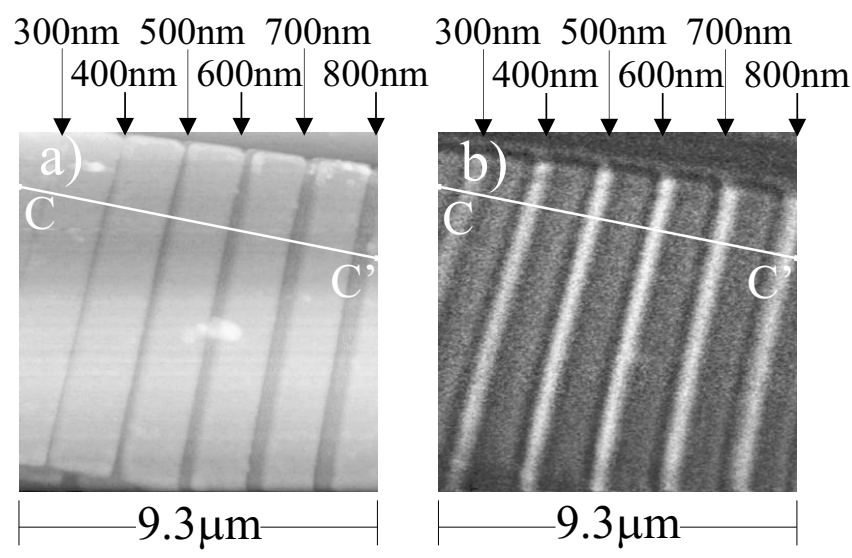




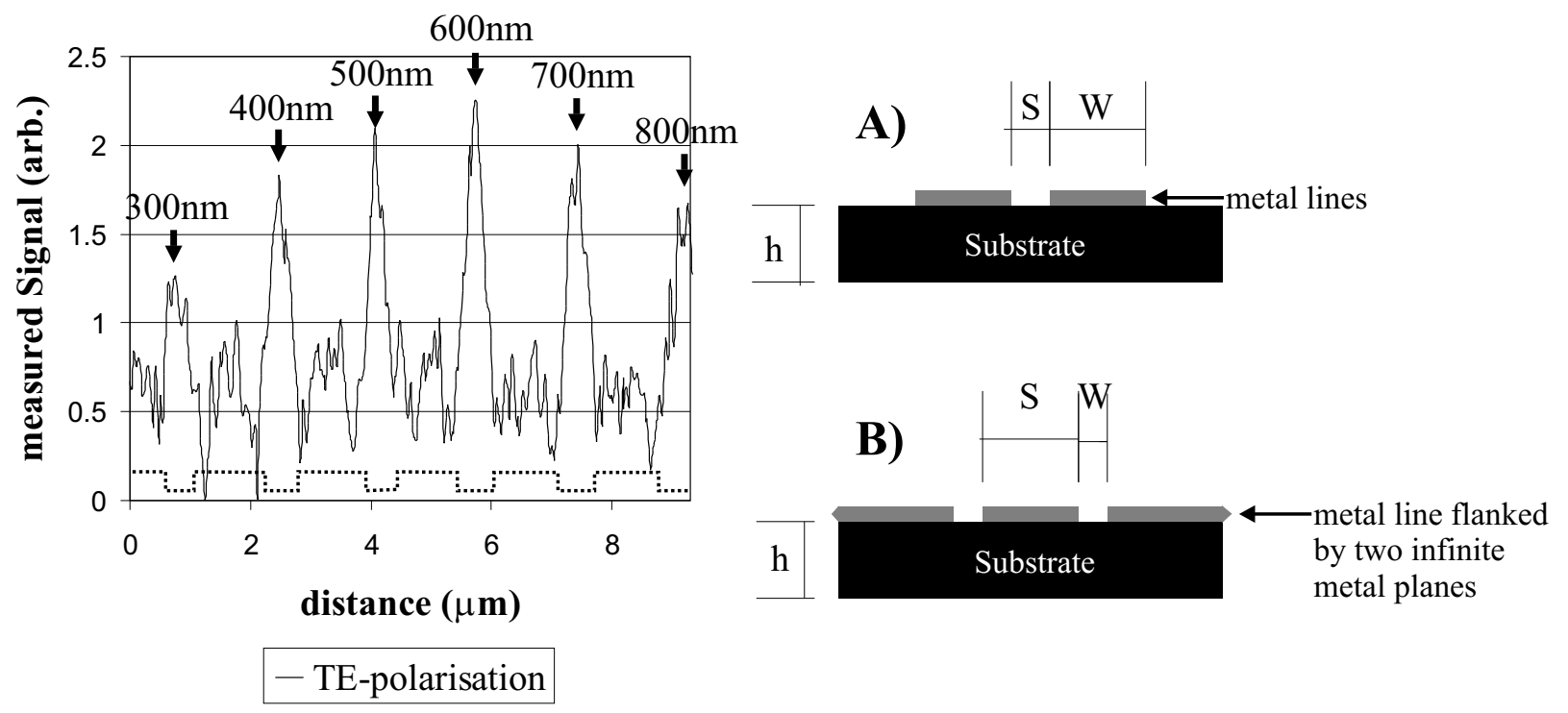

Figure 7

Figure 8 